\title{
Hosoya Polynomials of Steiner Distance of the Sequential Join of
} Graphs

Herish O. Abdullah

herish_omer@yahoo.com

College of Sciences

University of Salahaddin

Received on: 26/11/2007

Accepted on: 30/01/2008

ABSTRACT

The Hosoya polynomials of Steiner $\boldsymbol{n}$-distance of the sequential join of graphs $\boldsymbol{J}_{\mathbf{3}}$ and $\boldsymbol{J}_{\mathbf{4}}$ are obtained and the Hosoya polynomials of Steiner 3 distance of the sequential join of $\boldsymbol{m}$ graphs $\boldsymbol{J}_{\boldsymbol{m}}$ are also obtained.

Keywords: Steiner $\boldsymbol{n}$-distance, Hosoya polynomial, Sequential Join.

$$
\begin{aligned}
& \text { متعددات حدود هوسويا لمسافة ستينر -n لبيانات الجمع التتابعي } \\
& \text { هيرش عبدالله } \\
& \text { كلية العلوم، جامعة صلاح الدين }
\end{aligned}
$$

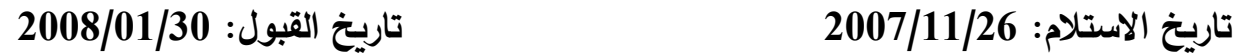

$$
\begin{aligned}
& \text { الملخص } \\
& \text { تضمن هذا البحث ايجاد متعددات حدود هوسويا لمسافة ستينر - } \\
& \text { التتابعي } \\
& \text { الك } \\
& \text { الكلمات المفتاحية: مسافة ستينر -n، متعددة حدود هوسويا، الجمع التتابعي. }
\end{aligned}
$$

\section{Introduction}

We follow the terminology of $[\mathbf{2 , 3}$ ]. For a connected graph $\boldsymbol{G}=(\boldsymbol{V}, \boldsymbol{E})$ of order $\boldsymbol{p}$, the Steiner distance $[\mathbf{5 , 6 , 7 ]}$ of a non-empty subset $S \subseteq V(G)$, denoted by $d_{G}(S)$, or simply $d(S)$, is defined to be the size of the smallest connected subgraph $T(S)$ of $G$ that contains $S ; T(S)$ is a tree called a Steiner tree of $S$. If $|S|=2$, then $d(S)$ is the distance between the two vertices of $\boldsymbol{S}$. For $2 \leq \boldsymbol{n} \leq \boldsymbol{p}$ and $|\boldsymbol{S}|=\boldsymbol{n}$, the Steiner distance of $\boldsymbol{S}$ is called Steiner $\boldsymbol{n}$-distance of $\boldsymbol{S}$ in $\boldsymbol{G}$. The Steiner $\boldsymbol{n}$-diameter of $\boldsymbol{G}$, denoted by $\operatorname{diam}_{n}^{*} \boldsymbol{G}$ or simply $\delta_{n}^{*}(\boldsymbol{G})$, is defined by:

$$
\operatorname{diam}_{n}^{*} G=\max \left\{d_{G}(S): S \subseteq V(G),|S|=n\right\} .
$$

Remark 1.1. It is clear that

(1) If $n>m$, then $\operatorname{diam}_{n}^{*} G \geq \operatorname{diam}_{m}^{*} G$.

(2) If $S^{\prime} \subseteq S$, then $d_{G}\left(S^{\prime}\right) \leq d_{G}(S)$. 
The Steiner $n$-distance of a vertex $v \in V(G)$, denoted by $W_{n}^{*}(v, G)$, is the sum of the Steiner $\boldsymbol{n}$-distances of all $\boldsymbol{n}$-subsets containing $\boldsymbol{v}$. The sum of Steiner $\boldsymbol{n}$-distances of all $\boldsymbol{n}$-subsets of $\boldsymbol{V}(\boldsymbol{G})$ is denoted by $\boldsymbol{d}_{\boldsymbol{n}}(\boldsymbol{G})$ or $\boldsymbol{W}_{\boldsymbol{n}}^{*}(\boldsymbol{G})$. It is clear that

$$
W_{n}^{*}(G)=n^{-1} \sum_{v \in V(G)} W_{n}^{*}(v, G) .
$$

The graph invariant $W_{n}^{*}(G)$ is called Wiener index of the Steiner n-distance of the graph $\boldsymbol{G}$.

Definition 1.2[1] Let $C_{n}^{*}(G, k)$ be the number of n-subsets of distinct vertices of $\boldsymbol{G}$ with Steiner $\boldsymbol{n}$-distance $\boldsymbol{k}$. The graph polynomial defined by

$$
\boldsymbol{H}_{n}^{*}(G ; \boldsymbol{x})=\sum_{k=n-1}^{\delta_{n}^{*}} \boldsymbol{C}_{n}^{*}(G, \boldsymbol{k}) \boldsymbol{x}^{k},
$$

where $\delta_{n}^{*}$ is the Steiner $\boldsymbol{n}$-diameter of $\boldsymbol{G}$; is called the Hosoya polynomial of Steiner n-distance of $G$.

It is clear that

$$
W_{n}^{*}(G)=\sum_{k=n-1}^{\delta_{n}^{*}} k C_{n}^{*}(G, k)
$$

For $1 \leq \boldsymbol{n} \leq \boldsymbol{p}$, let $\boldsymbol{C}_{\boldsymbol{n}}^{*}(\boldsymbol{u}, \boldsymbol{G}, \boldsymbol{k})$ be the number of $\boldsymbol{n}$-subsets $\boldsymbol{S}$ of distinct vertices of $\boldsymbol{G}$ containing $\boldsymbol{u}$ with Steiner $\boldsymbol{n}$-distance $\boldsymbol{k}$. It is clear that

$$
C_{1}^{*}(u, G, 0)=1 .
$$

Define

$$
\boldsymbol{H}_{n}^{*}(\boldsymbol{u}, \boldsymbol{G} ; \boldsymbol{x})=\sum_{k=n-1}^{\delta_{n}^{*}} \boldsymbol{C}_{n}^{*}(\boldsymbol{u}, \boldsymbol{G}, \boldsymbol{k}) \boldsymbol{x}^{k} .
$$

Obviously, for $2 \leq \boldsymbol{n} \leq \boldsymbol{p}$

$$
H_{n}^{*}(G ; x)=\frac{1}{n} \sum_{u \in V(G)} H_{n}^{*}(u, G ; x) .
$$

Ali and Saeed [1] were first who studied this distance-based graph polynomial for Steiner $\boldsymbol{n}$-distances, and established Hosoya polynomials of Steiner $\boldsymbol{n}$-distance for some special graphs and graphs having some kind of regularity, and for Gutman's compound graphs $\boldsymbol{G}_{\mathbf{1}} \bullet \boldsymbol{G}_{\mathbf{2}}$ and $\boldsymbol{G}_{\mathbf{1}}: \boldsymbol{G}_{\mathbf{2}}$ in terms of Hosoya polynomials of $\boldsymbol{G}_{\mathbf{1}}$ and $\boldsymbol{G}_{\mathbf{2}}$.

Definition 1.3[2] Let $\boldsymbol{G}_{\mathbf{1}}, \boldsymbol{G}_{\mathbf{2}}, \ldots, \boldsymbol{G}_{\boldsymbol{m}}, \boldsymbol{m} \geq \mathbf{2}$, be vertex disjoint graphs. The sequential join of $\boldsymbol{G}_{\mathbf{1}}, \boldsymbol{G}_{\mathbf{2}}, \ldots, \boldsymbol{G}_{\boldsymbol{m}}$ is a graph denoted by 


$$
J_{m}=G_{1}+G_{2}+\ldots+G_{m}
$$

and defined by

$$
\begin{aligned}
& \boldsymbol{V}\left(\boldsymbol{J}_{\boldsymbol{m}}\right)=\bigcup_{i=1}^{m} V\left(G_{i}\right) \\
& \left.E\left(J_{m}\right)=\left\{\bigcup_{i=1}^{m} E\left(G_{i}\right)\right\} \bigcup u v \mid u \in V_{i} \text { and } v \in V_{i+1}, \text { for } i=1,2, \ldots, m-1\right\}
\end{aligned}
$$

in which $\boldsymbol{V}_{\boldsymbol{i}}=\boldsymbol{V}\left(\boldsymbol{G}_{\boldsymbol{i}}\right)$, as depicted in the following figure.

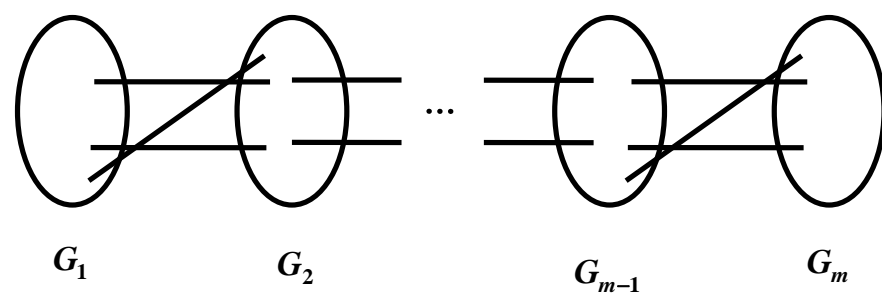

Fig. $1.1 J_{m}$

It is clear that

$$
p\left(J_{m}\right)=\sum_{i=1}^{m} p_{i}, q\left(J_{m}\right)=q_{m}+\sum_{i=1}^{m-1}\left(q_{i}+p_{i} p_{i+1}\right),
$$

in which

$$
p_{i}=p\left(G_{i}\right) \text { and } q_{i}=q\left(G_{i}\right) .
$$

One can easily see that for $\boldsymbol{m} \geq \mathbf{3}, \sum_{i=1}^{m} G_{i}$ is not commutative, that is for $m=3 \quad G_{1}+G_{2}+G_{3} \neq G_{1}+G_{3}+G_{2}$.

In [8], Saeed obtained the (ordinary) Hosoya polynomials of $\boldsymbol{J}_{\boldsymbol{m}}$, and in [7], Herish obtained the Steiner $\boldsymbol{n}$-diameter of the sequential join of $\boldsymbol{m}$ empty graphs and of $\boldsymbol{m}$ complete graphs. Also, the Hosoya polynomials of Steiner distance of the sequential join of $\boldsymbol{m}$ empty graphs and of $\boldsymbol{m}$ complete graphs were obtained. For $\boldsymbol{m} \geq \mathbf{3}$ and $\boldsymbol{n} \geq \mathbf{2}$, the Steiner $\boldsymbol{n}$-diameter of the sequential join of $\boldsymbol{m}$ complete graphs is given by[7]

$$
\operatorname{diam}_{n}^{*} J_{m}=\left\{\begin{array}{cl}
m+n-3, & \text { if } 2 \leq n \leq p_{1}+p_{m} \\
m+n-3-\alpha, & \text { if } p_{1}+p_{m}+1 \leq n \leq p,
\end{array}\right.
$$

where $\boldsymbol{\alpha}$ is the smallest integer such that 


$$
\boldsymbol{p}_{1}+\boldsymbol{p}_{\boldsymbol{m}}+1 \leq \boldsymbol{n} \leq \boldsymbol{p}_{1}+\boldsymbol{p}_{\boldsymbol{m}}+\sum_{i=1}^{\alpha} \boldsymbol{r}_{\boldsymbol{i}} .
$$

It is obvious that Eq. $\mathbf{1 . 6}$ holds for the sequential join of $\boldsymbol{m}$ graphs $\boldsymbol{J}_{\boldsymbol{m}}$.

In this paper, a generalization of the results obtained in [7] is given. We obtained the Hosoya polynomials of Steiner $\boldsymbol{n}$-distance of $\boldsymbol{J}_{\mathbf{3}}$ and $\boldsymbol{J}_{\mathbf{4}}$; and the Hosoya polynomials of Steiner 3-distance of $\boldsymbol{J}_{\boldsymbol{m}}, \boldsymbol{m} \geq \mathbf{4}$. We also obtained $\boldsymbol{H}_{n}^{*}\left(J_{3} ; \boldsymbol{x}\right)$, for $n \geq 2$ and $\boldsymbol{H}_{3}^{*}\left(J_{m} ; \boldsymbol{x}\right)$, for $\boldsymbol{m} \geq \mathbf{4}$, where each of $\boldsymbol{G}_{\boldsymbol{i}}$, for $i=1,2, \ldots, m$ is a special graph.

\section{Hosoya Polynomials of Steiner n-Distance of $J_{3}$ and $J_{4}$}

In this section, we consider $J_{m}$, for $\boldsymbol{m}=\mathbf{3}$ and $\boldsymbol{m}=\mathbf{4}$. Let $\boldsymbol{S}$ be any $\boldsymbol{n}$ subset of vertices of $\boldsymbol{J}_{\boldsymbol{m}}$. Let $\boldsymbol{B}\left(\boldsymbol{G}_{\boldsymbol{i}}\right)$, for $\boldsymbol{i}=\mathbf{1}, \mathbf{2}, \ldots, \boldsymbol{m}$, be the number of all $\boldsymbol{n}$ subsets $\boldsymbol{S}$ such that $\langle\boldsymbol{S}\rangle$ is connected in $\boldsymbol{G}_{\boldsymbol{i}}$. The following proposition determines the Hosoya polynomials of Steiner $\boldsymbol{n}$-distance of $\boldsymbol{J}_{\mathbf{3}}$.

Proposition 2.1. For $3 \leq n \leq p\left(=p_{1}+p_{2}+p_{3}\right)$,

$$
H_{n}^{*}\left(J_{3} ; x\right)=C_{1} x^{n-1}+C_{2} x^{n},
$$

where

$$
\begin{aligned}
& C_{1}=\left(\begin{array}{l}
p \\
n
\end{array}\right)-\left(\begin{array}{c}
p_{1}+p_{2} \\
n
\end{array}\right)-\left(\begin{array}{c}
p_{2} \\
n
\end{array}\right)+B\left(G_{1}\right)+B\left(G_{2}\right)+B\left(G_{3}\right), \\
& C_{2}=\left(\begin{array}{c}
p_{2} \\
n
\end{array}\right)+\left(\begin{array}{c}
p_{1}+p_{3} \\
n
\end{array}\right)-\left[B\left(G_{1}\right)+B\left(G_{2}\right)+B\left(G_{3}\right)\right]
\end{aligned}
$$

and

$$
\boldsymbol{B}\left(\boldsymbol{G}_{1}\right), \boldsymbol{B}\left(\boldsymbol{G}_{2}\right) \text { and } \boldsymbol{B}\left(\boldsymbol{G}_{3}\right) \text { are as defined above. }
$$

Proof. It is clear that

Therefore,

$$
\text { diam }_{n}^{*} J_{3}=\left\{\begin{array}{ccc}
n, & \text { if } & 3 \leq n \leq p_{1}+p_{3} \\
n-1, & \text { if } & \text { otherwise }
\end{array} .\right.
$$

$$
H_{n}^{*}\left(J_{3} ; x\right)=C_{1} x^{n-1}+C_{2} x^{n}
$$

in which $C_{1}$ is the number of all $n$-subsets of $V\left(J_{3}\right)$ with Steiner distance equals $n-1$, and $C_{2}$ is the number of all $n$-subsets of $V\left(J_{3}\right)$ with Steiner distance equals $\boldsymbol{n}$.

Therefore,

$$
C_{2}=\sum_{i=1}^{3}\left\{\left(\begin{array}{c}
p_{i} \\
n
\end{array}\right)-B\left(G_{i}\right)\right\}+\sum_{j=1}^{n-1}\left(\begin{array}{c}
p_{1} \\
j
\end{array}\right)\left(\begin{array}{c}
p_{3} \\
n-j
\end{array}\right)
$$




$$
=\left(\begin{array}{c}
p_{2} \\
n
\end{array}\right)+\left(\begin{array}{c}
p_{1}+p_{3} \\
n
\end{array}\right)-\left[B\left(G_{1}\right)+B\left(G_{2}\right)+B\left(G_{3}\right)\right]
$$

Now, since

therefore

$$
\boldsymbol{C}_{1}+\boldsymbol{C}_{2}=\left(\begin{array}{l}
\boldsymbol{p} \\
\boldsymbol{n}
\end{array}\right)
$$

$$
C_{1}=\left(\begin{array}{l}
p \\
n
\end{array}\right)-C_{2}=\left(\begin{array}{l}
p \\
n
\end{array}\right)-\left(\begin{array}{c}
p_{1}+p_{3} \\
n
\end{array}\right)-\left(\begin{array}{c}
p_{2} \\
n
\end{array}\right)+B\left(G_{1}\right)+B\left(G_{2}\right)+B\left(G_{3}\right)
$$

This completes the proof.

The following corollary computes the $\boldsymbol{n}$-Wiener index of $\boldsymbol{J}_{\mathbf{3}}$.

Corollary 2.2. For $3 \leq n \leq p\left(=p_{1}+p_{2}+p_{3}\right)$,

$$
W_{n}^{*}\left(J_{3}\right)=n\left(\begin{array}{l}
p \\
n
\end{array}\right)-C_{1},
$$

where $\boldsymbol{C}_{\mathbf{1}}$ is given in Proposition 2.1.

Next, we shall find the Hosoya polynomials of Steiner $\boldsymbol{n}$-distance of $\boldsymbol{J}_{\mathbf{4}}$.

Proposition 2.3. For $3 \leq n \leq p\left(=p_{1}+p_{2}+p_{3}+p_{4}\right)$,

where

$$
H_{n}^{*}\left(J_{4} ; x\right)=C_{1} x^{n-1}+C_{2} x^{n}+C_{3} x^{n+1},
$$

$$
\begin{aligned}
C_{1}= & \sum_{i=1}^{n-2} \sum_{j=1}^{n-1-i}\left[\left(\begin{array}{c}
p_{1} \\
i
\end{array}\right)\left(\begin{array}{c}
p_{2} \\
j
\end{array}\right)\left(\begin{array}{c}
p_{3} \\
n-i-j
\end{array}\right)+\left(\begin{array}{c}
p_{2} \\
i
\end{array}\right)\left(\begin{array}{c}
p_{3} \\
j
\end{array}\right)\left(\begin{array}{c}
p_{4} \\
n-i-j
\end{array}\right)\right] \\
& +\sum_{i=1}^{n-3} \sum_{j=1}^{n-2-i} \sum_{k=1}^{n-1-i-j}\left(\begin{array}{c}
p_{1} \\
i
\end{array}\right)\left(\begin{array}{c}
p_{2} \\
j
\end{array}\right)\left(\begin{array}{c}
p_{3} \\
k
\end{array}\right)\left(\begin{array}{c}
p_{4} \\
n-i-j-k
\end{array}\right)+\sum_{i=1}^{4} B\left(G_{i}\right) \\
& +\left(\begin{array}{c}
p_{1}+p_{2} \\
n
\end{array}\right)+\left(\begin{array}{c}
p_{2}+p_{3} \\
n
\end{array}\right)+\left(\begin{array}{c}
p_{3}+p_{4} \\
n
\end{array}\right)-\left(\begin{array}{c}
p_{1} \\
n
\end{array}\right)-2\left(\begin{array}{c}
p_{2} \\
n
\end{array}\right)-2\left(\begin{array}{c}
p_{3} \\
n
\end{array}\right)-\left(\begin{array}{c}
p_{4} \\
n
\end{array}\right), \\
C_{2}= & \left(\begin{array}{c}
p \\
n
\end{array}\right)-\sum_{i=1}^{n-2} \sum_{j=1}^{n-1-i}\left[\left(\begin{array}{c}
p_{1} \\
i
\end{array}\right)\left(\begin{array}{c}
p_{2} \\
j
\end{array}\right)\left(\begin{array}{c}
p_{3} \\
n-i-j
\end{array}\right)\right] \\
& -\sum_{i=1}^{n-3} \sum_{j=1}^{n-2-i} \sum_{k=1}^{n-1-i-j}\left(\begin{array}{c}
p_{1} \\
i
\end{array}\right)\left(\begin{array}{c}
p_{2} \\
j
\end{array}\right)\left(\begin{array}{c}
p_{3} \\
k
\end{array}\right)\left(\begin{array}{c}
n-i-j-k \\
n-1
\end{array}\right)-\sum_{i=1}^{4} B\left(G_{i}\right) \\
& -\left(\begin{array}{c}
p_{1}+p_{2} \\
n
\end{array}\right)-\left(\begin{array}{c}
p_{2}+p_{3} \\
n
\end{array}\right)-\left(\begin{array}{c}
p_{3}+p_{4} \\
n
\end{array}\right)-\left(\begin{array}{c}
p_{1}+p_{4} \\
n
\end{array}\right)
\end{aligned}
$$




$$
+2\left(\begin{array}{c}
p_{1} \\
n
\end{array}\right)+2\left(\begin{array}{c}
p_{2} \\
n
\end{array}\right)+2\left(\begin{array}{c}
p_{3} \\
n
\end{array}\right)+2\left(\begin{array}{c}
p_{4} \\
n
\end{array}\right)
$$

and

$$
C_{3}=\left(\begin{array}{c}
p_{1}+p_{4} \\
n
\end{array}\right)-\left(\begin{array}{c}
p_{1} \\
n
\end{array}\right)-\left(\begin{array}{c}
p_{4} \\
n
\end{array}\right) \text {. }
$$

Proof. It is clear that $n-\mathbf{1} \leq \operatorname{diam}_{n}^{*} J_{\mathbf{4}} \leq \boldsymbol{n + 1}$, therefore the Hosoya polynomials of Steiner $\boldsymbol{n}$-distance of $\boldsymbol{J}_{\mathbf{4}}$ has the following form

$$
H_{n}^{*}\left(J_{4} ; x\right)=C_{1} x^{n-1}+C_{2} x^{n}+C_{3} x^{n+1} .
$$

To find $\boldsymbol{C}_{1}, \boldsymbol{C}_{2}$ and $\boldsymbol{C}_{3}$, let $\boldsymbol{S}$ be any $\boldsymbol{n}$-subset of vertices of $\boldsymbol{J}_{\mathbf{4}}$, then we have the following possibilities for the subset $S$.

(I) $\boldsymbol{d}(\boldsymbol{S})=\boldsymbol{n}-\mathbf{1}$ if and only if $\mathrm{S}$ has any of the following subcases:

(1) $S$ is a subset of $V_{i}$, for $i=1,2,3,4$ and $\langle S\rangle$ is a connected subgraph of $\boldsymbol{G}_{\boldsymbol{i}}$. The number of these $\boldsymbol{n}$-subsets is given by

$$
B\left(G_{1}\right)+B\left(G_{2}\right)+B\left(G_{3}\right)+B\left(G_{4}\right) \text {. }
$$

(2) $S \subseteq V_{k} \cup V_{k+1}$ and $\left(S \cap V_{k} \neq \varphi \wedge S \cap V_{k+1} \neq \varphi\right), k=1,2,3$.

The number of these subsets $S$ is given by

$$
\begin{aligned}
& \sum_{i=1}^{n-1}\left(\begin{array}{c}
p_{1} \\
i
\end{array}\right)\left(\begin{array}{c}
p_{2} \\
n-i
\end{array}\right)+\sum_{i=1}^{n-1}\left(\begin{array}{c}
p_{2} \\
i
\end{array}\right)\left(\begin{array}{c}
p_{3} \\
n-i
\end{array}\right)+\sum_{i=1}^{n-1}\left(\begin{array}{c}
p_{3} \\
i
\end{array}\right)\left(\begin{array}{c}
p_{4} \\
n-i
\end{array}\right) \\
& =\left(\begin{array}{c}
p_{1}+p_{2} \\
n
\end{array}\right)+\left(\begin{array}{c}
p_{2}+p_{3} \\
n
\end{array}\right)+\left(\begin{array}{c}
p_{3}+p_{4} \\
n
\end{array}\right)-\left(\begin{array}{l}
p_{1} \\
n
\end{array}\right)-2\left(\begin{array}{c}
p_{2} \\
n
\end{array}\right)-2\left(\begin{array}{c}
p_{3} \\
n
\end{array}\right)-\left(\begin{array}{c}
p_{4} \\
n
\end{array}\right),
\end{aligned}
$$

(3) $\left(S \subseteq \bigcup_{i=1}^{3} V_{i} \wedge S \cap V_{i} \neq \varphi\right)$ or $\left(S \subseteq \bigcup_{i=2}^{4} V_{i} \wedge S \cap V_{i} \neq \varphi\right)$. The number of these $\boldsymbol{n}$-subsets is given by

$$
\sum_{i=1}^{n-2} \sum_{j=1}^{n-1-i}\left[\left(\begin{array}{c}
p_{1} \\
i
\end{array}\right)\left(\begin{array}{c}
p_{2} \\
j
\end{array}\right)\left(\begin{array}{c}
p_{3} \\
n-i-j
\end{array}\right)+\left(\begin{array}{c}
p_{2} \\
i
\end{array}\right)\left(\begin{array}{c}
p_{3} \\
j
\end{array}\right)\left(\begin{array}{c}
p_{4} \\
n-i-j
\end{array}\right)\right]
$$

(4) $S \cap V_{i} \neq \varphi, i=1,2,3,4$. The number of these $n$-subsets is given by

$$
\sum_{i=1}^{n-3} \sum_{j=1}^{n-2-i} \sum_{k=1}^{n-1-i-j}\left(\begin{array}{c}
p_{1} \\
i
\end{array}\right)\left(\begin{array}{c}
p_{2} \\
j
\end{array}\right)\left(\begin{array}{c}
p_{3} \\
k
\end{array}\right)\left(\begin{array}{c}
p_{4} \\
n-i-j-k
\end{array}\right)
$$

From (1), (2), (3) and (4), we get $\boldsymbol{C}_{\mathbf{1}}$ as given in the statement of the proposition.

(II) $d(S)=n+1$ if and only if $S \subseteq V_{1} \cup V_{4}$ and $\left(S \cap V_{1} \neq \varphi\right.$ and $S \cap V_{4} \neq \varphi$ ). The number of these $S$ 's is given by 
$\sum_{i=1}^{n-1}\left(\begin{array}{c}p_{1} \\ i\end{array}\right)\left(\begin{array}{c}p_{4} \\ n-i\end{array}\right)=\left(\begin{array}{c}p_{1}+p_{4} \\ n\end{array}\right)-\left(\begin{array}{c}p_{1} \\ n\end{array}\right)-\left(\begin{array}{c}p_{4} \\ n\end{array}\right)$

So, $\boldsymbol{C}_{\mathbf{3}}$ is as given.

Now, since $C_{1}+C_{2}+C_{3}=\left(\begin{array}{l}p \\ n\end{array}\right)$,

therefore

$$
C_{2}=\left(\begin{array}{l}
p \\
n
\end{array}\right)-C_{1}-C_{3}
$$

This completes the proof.

Remark. The triple summation in $\boldsymbol{C}_{\mathbf{1}}$ is taken to be zero when $\boldsymbol{n = 3}$.

The following corollary computes $\boldsymbol{W}_{n}^{*}\left(\boldsymbol{J}_{4}\right)$.

Corollary 2.4. For $3 \leq n \leq p\left(=p_{1}+p_{2}+p_{3}+p_{4}\right)$,

$$
W_{n}^{*}\left(J_{4}\right)=n\left(\begin{array}{l}
p \\
n
\end{array}\right)-C_{1}+C_{3},
$$

where $\boldsymbol{C}_{\mathbf{1}}$ and $\boldsymbol{C}_{\mathbf{3}}$ are given in Proposition 2.3.

Remark. For $\boldsymbol{m} \geq \mathbf{5}$, the calculation of the coefficients of $\boldsymbol{H}_{n}^{*}\left(\boldsymbol{J}_{\boldsymbol{m}} ; \boldsymbol{x}\right)$ is complicated.

The numbers $\boldsymbol{B}\left(\boldsymbol{G}_{\mathbf{1}}\right), \boldsymbol{B}\left(\boldsymbol{G}_{\mathbf{2}}\right)$ and $\boldsymbol{B}\left(\boldsymbol{G}_{3}\right)$ are given in Proposition 2.1 can be counted for some specific graphs $\boldsymbol{G}_{\mathbf{1}}, \boldsymbol{G}_{\mathbf{2}}$ and $\boldsymbol{G}_{\mathbf{3}}$ as in the following examples.

Example 2.5. Let $N_{p_{1}}, N_{p_{2}}$ and $N_{p_{3}}$ be empty graphs of orders $\boldsymbol{p}_{1}, \boldsymbol{p}_{2}$ and $\boldsymbol{p}_{3}$ respectively, then

$$
B\left(N_{p_{1}}\right)=B\left(N_{p_{2}}\right)=B\left(N_{p_{3}}\right)=0 .
$$

Example 2.6. Let $\boldsymbol{K}_{p_{1}}, \boldsymbol{K}_{p_{2}}$ and $\boldsymbol{K}_{p_{3}}$ be complete graphs of orders $\boldsymbol{p}_{\mathbf{1}}, \boldsymbol{p}_{\mathbf{2}}$ and $\boldsymbol{p}_{\mathbf{3}}$ respectively, then

$$
B\left(K_{p_{i}}\right)=\left(\begin{array}{c}
p_{i} \\
n
\end{array}\right), \text { for } i=1,2,3 .
$$

Example 2.7. Let $\boldsymbol{P}_{\alpha_{1}}, \boldsymbol{P}_{\alpha_{2}}$ and $\boldsymbol{P}_{\alpha_{3}}$ be path graphs of orders $\boldsymbol{\alpha}_{1}, \boldsymbol{\alpha}_{2}$ and $\boldsymbol{\alpha}_{3}$ respectively, then

$$
B\left(P_{\alpha_{i}}\right)=\alpha_{i}-n+1 \text {, for } i=1,2,3 \text {. }
$$


Example 2.8. Let $C_{\alpha_{1}}, C_{\alpha_{2}}$ and $C_{\alpha_{3}}$ be cycle graphs of orders $\boldsymbol{\alpha}_{1}, \boldsymbol{\alpha}_{2}$ and $\alpha_{3}$ respectively, then

$$
B\left(C_{\alpha_{i}}\right)=\alpha_{i}, \text { for } i=1,2,3 \text {. }
$$

Example 2.9. Let $\boldsymbol{W}_{\alpha_{1}}, \boldsymbol{W}_{\alpha_{2}}$ and $\boldsymbol{W}_{\alpha_{3}}$ be wheel graphs of orders $\boldsymbol{\alpha}_{1}, \boldsymbol{\alpha}_{2}$ and $\alpha_{3}$ respectively, then

$$
B\left(W_{\alpha_{i}}\right)=\left(\begin{array}{c}
\alpha_{i}-1 \\
n-1
\end{array}\right)+\alpha_{i}-1, \text { for } i=1,2,3 .
$$

Example 2.10. Let $K_{\alpha_{i}, \beta_{i}}$, for $\boldsymbol{i}=\mathbf{1 , 2 , 3}$, be complete bipartite graphs of partite sets of size $\alpha_{i} \beta_{i}$, then

$$
B\left(K_{\alpha_{i}, \beta_{i}}\right)=\left(\begin{array}{c}
\alpha_{i}+\beta_{i} \\
n
\end{array}\right)-\left(\begin{array}{c}
\alpha_{i} \\
n
\end{array}\right)-\left(\begin{array}{c}
\beta_{i} \\
n
\end{array}\right), \text { for } i=1,2,3 .
$$

\section{Hosoya Polynomials of Steiner 3-Distance of $\boldsymbol{J}_{\boldsymbol{m}}(\boldsymbol{m} \geq \mathbf{5})$}

In this section, we consider $\boldsymbol{J}_{\boldsymbol{m}}=\boldsymbol{G}_{\mathbf{1}}+\boldsymbol{G}_{\mathbf{2}}+\ldots+\boldsymbol{G}_{\boldsymbol{m}}$, for $\boldsymbol{m} \geq \mathbf{5}$. The following theorem determines Hosoya polynomials of Steiner 3-distance of $J_{m}$.

Theorem 3.1. For $m \geq 5$,

$$
\begin{aligned}
H_{3}^{*}\left(J_{m} ; x\right) & =(A+B x) x^{2}+\frac{1}{2} \sum_{j=i+1}^{m} \sum_{i=1}^{m-1} p_{i} p_{j}\left(p_{i}+p_{j}-2\right) x^{j-i+1} \\
& +\sum_{j=i+2}^{m} \sum_{i=1}^{m-2} p_{i} p_{j}\left(\sum_{r=1}^{j-1} p_{r}\right) x^{j-i}
\end{aligned}
$$

where

$$
A=\sum_{i=1}^{m}\left[\sum_{v \in V_{i}}\left(\begin{array}{c}
\operatorname{deg} v \\
2
\end{array}\right)-2 \gamma_{i}\right], B=\sum_{i=1}^{m}\left(\begin{array}{c}
p_{i} \\
3
\end{array}\right)-A,
$$

in which $\gamma_{\boldsymbol{i}}$, for $\boldsymbol{i}=\mathbf{1 , 2}, \ldots, \boldsymbol{m}$ is the number of non-identical triangles $\boldsymbol{K}_{\mathbf{3}}$ as a subgraph in $\boldsymbol{G}_{\boldsymbol{i}}$.

Proof. Let $S$ be any 3 -subset of vertices of $\boldsymbol{J}_{\boldsymbol{m}}$, then we have three main cases for the subset $\boldsymbol{S}$.

(I) If $S \subseteq V_{i}$, for $i=1,2, \ldots, m$, then

(a) $d(S)=2$, when $\langle S\rangle$ is a connected subgraph in $\boldsymbol{G}_{i}$, and by Lemma 3.4.4. of [7], the number of such 3 -subsets $S$ is given by 


$$
A=\sum_{i=1}^{m}\left[\sum_{v \in V_{i}}\left(\begin{array}{c}
\operatorname{deg} v \\
2
\end{array}\right)-2 \gamma_{i}\right] .
$$

(b) $\boldsymbol{d}(\boldsymbol{S})=\mathbf{3}$, when $\langle\boldsymbol{S}\rangle$ is a disconnected subgraph in $\boldsymbol{G}_{\boldsymbol{i}}$, and the number of such 3 -subsets $S$ is given by

$$
B=\sum_{i=1}^{m}\left(\begin{array}{c}
p_{i} \\
3
\end{array}\right)-A \text {. }
$$

Case $(\mathbf{I})$ produces the polynomial

$$
F_{1}(x)=(A+B x) x^{2} .
$$

(II) Either two vertices of $\mathrm{S}$ are in $\boldsymbol{V}_{\boldsymbol{i}}$ and one vertex of $\boldsymbol{S}$ in $\boldsymbol{V}_{\boldsymbol{j}}, \boldsymbol{i}<\boldsymbol{j}$, or one vertex of $S$ in $V_{i}$, and two vertices of $S$ in $V_{j}$, for $1 \leq i<j \leq m$. For each such cases of $\boldsymbol{S}$,

$$
d(S)=j-i+1,
$$

and the number of ways of choosing such $S$ is given by

$$
\sum_{j=i+1}^{m} \sum_{i=1}^{m-1}\left[\left(\begin{array}{c}
\boldsymbol{p}_{\boldsymbol{i}} \\
2
\end{array}\right) \boldsymbol{p}_{j}+\left(\begin{array}{c}
\boldsymbol{p}_{j} \\
2
\end{array}\right) \boldsymbol{p}_{\boldsymbol{i}}\right],
$$

and, this produces the polynomial

$$
\begin{aligned}
\boldsymbol{F}_{2}(x) & =\frac{1}{2} \sum_{j=i+1}^{m} \sum_{i=1}^{m-1}\left[\boldsymbol{p}_{j} \boldsymbol{p}_{i}\left(\boldsymbol{p}_{i}-1\right)+\boldsymbol{p}_{i} \boldsymbol{p}_{j}\left(\boldsymbol{p}_{j}-1\right)\right] x^{j-i+1} \\
& =\frac{1}{2} \sum_{j=i+1}^{m} \sum_{i=1}^{m-1} \boldsymbol{p}_{i} \boldsymbol{p}_{j}\left(\boldsymbol{p}_{i}+\boldsymbol{p}_{j}-2\right) x^{j-i+1}
\end{aligned}
$$

(III) One vertex of $\mathrm{S}$ in $\boldsymbol{V}_{\boldsymbol{i}}$, one vertex in $\boldsymbol{V}_{j}, \boldsymbol{j} \geq \boldsymbol{i}+\mathbf{2}$, and the third vertex in $V_{r}, \boldsymbol{i}<\boldsymbol{r}<\boldsymbol{j}$. For such case

$$
d(S)=j-i
$$

and the number of all possibilities of such $S$ is

$$
\sum_{j=i+2}^{m} \sum_{i=1}^{m-2} p_{i} p_{j}\left(\sum_{r=i+1}^{j-1} p_{r}\right),
$$

and this produces the polynomial

$$
\boldsymbol{F}_{3}(\boldsymbol{x})=\sum_{j=i+2}^{m} \sum_{i=1}^{m-2} \boldsymbol{p}_{i} \boldsymbol{p}_{j}\left(\sum_{r=i+1}^{j-1} \boldsymbol{p}_{\boldsymbol{r}}\right) \boldsymbol{x}^{j-i} .
$$

Now adding the polynomials $\boldsymbol{F}_{1}(\boldsymbol{x}), \boldsymbol{F}_{2}(\boldsymbol{x})$ and $\boldsymbol{F}_{3}(\boldsymbol{x})$ obtained in (I), (II) and (III), we get the required result.

The numbers $\boldsymbol{A}$ and $\boldsymbol{B}$ are given in Theorem 3.1 can be counted when $\boldsymbol{G}_{\boldsymbol{i}}$, for $\boldsymbol{i}=\mathbf{1}, \mathbf{2}, \ldots, \boldsymbol{m}$, has a special form, as in the following examples. 
Example 3.2. Let $N_{p_{i}}$, for $i=\mathbf{1 , 2}, \ldots, m$ be empty graphs of orders $p_{i}$, then

$$
\boldsymbol{A}=\mathbf{0} \text { and } \boldsymbol{B}=\sum_{i=1}^{m}\left(\begin{array}{c}
p_{i} \\
3
\end{array}\right)
$$

Example 3.3. Let $K_{p_{i}}$, for $i=1,2, \ldots, m$ be complete graphs of orders $\boldsymbol{p}_{i}$, then

$$
\boldsymbol{A}=\sum_{i=1}^{m}\left(\begin{array}{c}
\boldsymbol{p}_{\boldsymbol{i}} \\
\mathbf{3}
\end{array}\right) \text { and } \boldsymbol{B}=\mathbf{0}
$$

Example 3.4. Let $\boldsymbol{P}_{\alpha_{i}}$, for $\boldsymbol{i}=\mathbf{1 , 2}, \ldots, \boldsymbol{m}$ be path graphs of orders $\boldsymbol{\alpha}_{i}$, then

$$
A=\sum_{i=1}^{m}\left[\alpha_{i}-2\right]=p-2 m \text { and } B=\sum_{i=1}^{m}\left(\begin{array}{c}
\alpha_{i} \\
3
\end{array}\right)-p+2 m \text {. }
$$

Example 3.5. Let $C_{\alpha_{i}}$, for $i=1,2, \ldots, m$ be cycle graphs of orders $\alpha_{i}$, then

$$
\boldsymbol{A}=\sum_{i=1}^{m} \alpha_{i}=p \text { and } B=\sum_{i=1}^{m}\left(\begin{array}{c}
\alpha_{i} \\
3
\end{array}\right)-p
$$

Example 3.6. Let $\boldsymbol{W}_{\alpha_{i}}$ for $\boldsymbol{i}=\mathbf{1 , 2}, \ldots, \boldsymbol{m}$ be wheel graphs of orders $\boldsymbol{\alpha}_{i}$, then

$$
\begin{aligned}
A & =\sum_{i=1}^{m}\left[\sum_{v \in V_{i}}\left(\begin{array}{c}
\operatorname{deg} v \\
2
\end{array}\right)-2 \gamma_{i}\right]=\sum_{i=1}^{m}\left[\left(\alpha_{i}-1\right)\left(\begin{array}{l}
3 \\
2
\end{array}\right)+\left(\begin{array}{c}
\alpha_{i}-1 \\
2
\end{array}\right)-2\left(\alpha_{i}-1\right)\right] \\
& =\sum_{i=1}^{m}\left(\begin{array}{c}
\alpha_{i} \\
2
\end{array}\right)
\end{aligned}
$$

and

$$
B=\sum_{i=1}^{m}\left(\begin{array}{c}
\alpha_{i} \\
3
\end{array}\right)-\sum_{i=1}^{m}\left(\begin{array}{c}
\alpha_{i} \\
2
\end{array}\right)=\frac{1}{6} \sum_{i=1}^{m} \alpha_{i}\left(\alpha_{i}-1\right)\left(\alpha_{i}-5\right) .
$$

Example 3.7. Let $K_{\alpha_{i}, \beta_{i}}$, for $i=1,2, \ldots, m$, be complete bipartite graphs of partite sets of size $\boldsymbol{\alpha}_{i} \boldsymbol{\beta}_{i}$, then it is known that $\boldsymbol{K}_{\boldsymbol{\alpha}_{i}, \beta_{i}}$ contains no odd cycles, and so $\gamma_{i}=\mathbf{0}$, for $i=1,2, \ldots, m$.

Hence,

$$
A=\sum_{i=1}^{m}\left[\alpha_{i}\left(\begin{array}{c}
\beta_{i} \\
2
\end{array}\right)+\beta_{i}\left(\begin{array}{c}
\alpha_{i} \\
2
\end{array}\right)\right]=\frac{1}{2} \sum_{i=1}^{m} \alpha_{i} \beta_{i}\left(\alpha_{i}+\beta_{i}-2\right),
$$

and

$$
B=\sum_{i=1}^{m}\left[\left(\begin{array}{c}
\alpha_{i}+\beta_{i} \\
3
\end{array}\right)-\frac{1}{2} \alpha_{i} \beta_{i}\left(\alpha_{i}+\beta_{i}-2\right)\right]
$$




\section{REFERENCES}

[1] Ali, A.A. and Saeed, W.A; (2006), "Wiener polynomials of Steiner distance of graphs", J. of Applied Sciences, Vol.8, No.2, pp.64-71.

[2] Buckly, F. and Harary, F.;(1990), Distance in Graphs, AddisonWesley, Redwood, California.

[3] Chartrand, G. and Lesniak, L.; (1986), Graphs and Digraphs, $2^{\text {nd }}$ ed., Wadsworth and Brooks/ Cole, California.

[4] Danklemann, P., Swart, H.C. and Oellermann, O.R.; (1997), "On the average Steiner distance of graphs with prescribed properties", Discrete Applied Maths., Vol.79, pp.91-103.

[5] Danklemann, P., Oellermann, O. R. and Swart, H.C.; (1996), “ The average Steiner distance of a graph", J. Graph Theory, Vol.22, No.1, pp.15-22.

[6] Henning, M.A., Oellermann, O.R., and Swart, H.C.;(1991), "On vertices with maximum Steiner eccentricity in graphs", Graph Theory, Combinatorics, Algorithms and Applications (eds. Y. Alavi, F.R.K. Chung, R.L. Graham, and D.F. Hsu.), SIAM publication, Philadelphia, pp.393-403.

[7] Herish, O.A.; (2007), Hosoya Polynomials of Steiner Distance of Some Graphs, Ph.D. thesis, Salahaddin University, Erbil.

[8] Saeed, W.A.M.; (1999), Wiener Polynomials of Graphs, Ph.D. thesis, Mosul University, Mosul. 